\title{
A Negotiating Agents Model for the Provision of Flexible Telephony Services
}

\author{
M. Rizzo \\ Dept. of Computer Science \& A.I. \\ University of Malta \\ Msida MSD 13 \\ Malta
}

\author{
I. A. Utting \\ Computing Laboratory \\ University of Kent \\ Canterbury, Kent CT2 7NF \\ England
}

\begin{abstract}
Current telephone systems suffer from a service interface bottleneck problem, wherein network resources are under-utilised and customer requirements are often not met, in spite of these resources' ability to satisfy such requirements. This bottleneck is primarily due to a coarse-grain service interface, coupled with the inability to support arbitrary terminal types, and the inability to inter-operate with other systems.

This paper outlines a new model for telephony services, based on the concept of negotiating agents. In this model, functionality is not made available to users in the form of services, as has been the case traditionally. Instead, users specify policies that describe how they wish their calls to be handled. These policies are used to guide agents appointed to act on behalf of users. The paper also describes a prototype that was built to demonstrate the capabilities of the model.
\end{abstract}

\section{Introduction}

Developments in telecommunications technology have, in recent years, enabled network operators to introduce a range of supplementary services. These first appeared as extensions to the traditional Public Switched Telephone Network (PSTN), then in the Integrated Services Digital Network (ISDN), and more recently in the Intelligent Network (IN).

Supplementary services modify the normal call setup process to provide some useful function. Popular examples are Freephone, Call Forwarding on Busy, and Call Waiting. In Call Forwarding on Busy, for instance, call setup is modified so that if the destination number is busy, the call is routed to another number.

Many supplementary services allow some degree of customisation, thereby enabling subscribers to tailor services to their specific needs. For example, Call Forwarding on Busy allows subscribers to specify a forwarding number. However, the extent to which such services may be customised is generally rather lim- ited, offering little expressive power to the subscriber, who is only allowed to specify values to service parameters and turn services on and off. Consequently, these services cannot always meet customer demands, even if network resources could, in principle, be used to provide the required functionality. This problem is termed the service interface bottleneck.

This paper describes NAT (Negotiating Agents for Telephony), an alternative model for the provision of telephony services which aims to deliver increased functionality to end users. Rather than package functionality into a set of rigid, inflexible services, NAT allows users to express policies which describe how they would like their calls to be managed. These policies are used to guide users' agents, which negotiate and take action on behalf of their users. The aim of negotiation is to find a suitable course of action that is acceptable to all users concerned.

The paper is structured as follows: section 2 describes the service interface bottleneck, showing how it is related to the well-known problem of feature interaction [1]; section 3 reviews relevant research activities; section 4 introduces the NAT model; section 5 describes a prototype system that was built to demonstrate the capabilities of the model; section 6 concludes with an evaluation of the NAT approach.

\section{The service interface bottleneck}

Consider a telephone user $X$ who wishes to have all his/her calls forwarded to a user $Y$ if $Y$ is not busy at the time of the call, or to another user $Z$ otherwise. Clearly, a stored program control exchange is capable of doing this, and any necessary control messages could easily be carried over a modern digital transmission signalling network. Yet, such a service is not defined for PSTN and ISDN supplementary services, nor is it listed in existing IN capability sets.

Unfortunately, the problem highlighted by this simple example is characteristic of today's telephone sys- 